\title{
AN INVENTORY-ROUTING PROBLEM WITH STOCHASTIC DEMAND AND STOCK-OUT: A SOLUTION AND RISK ANALYSIS USING SIMHEURISTICS
}

\author{
Bhakti Stephan Onggo \\ CORMSIS - Southampton Business School \\ University of Southampton \\ University Road \\ Southampton, SO17 1BJ, UK
}

\author{
Angel A. Juan \\ Javier Panadero \\ Universitat Oberta de Catalunya \\ Euncet Business School \\ Av. Carl Friedrich Gauss 5 \\ Castelldefels, 08860, SPAIN
}

\author{
Canan G. Corlu \\ Metropolitan College \\ Boston University \\ 1010 Commonwealth Avenue \\ Boston, MA, 02215, USA
}

\author{
Alba Agustin \\ Dept. of Statistics, Informatics, and Mathematics \\ Public University of Navarre \\ Campus de Arrosadia \\ Pamplona, 31006, SPAIN
}

\begin{abstract}
Supply chain operations have become more complex. Hence, in order to optimise supply chain operations, we often need to simplify the optimisation problem in such a way that it can be solved efficiently using either exact methods or metaheuristics. One common simplification is to assume all model inputs are deterministic. However, for some management decisions, considering the uncertainty in model inputs (e.g., demands, travel times, processing times) is essential. Otherwise, the results may be misleading and might lead to an incorrect decision. This paper considers an example of a complex supply chain operation that can be viewed as an Inventory-Routing Problem with stochastic demands. We demonstrate how a simheuristic framework can be employed to solve the problem. Further, we illustrate the risks of not considering input uncertainty. The results show that simheuristics can produce a good result, and ignoring the uncertainty in the model input may lead to sub-optimal results.
\end{abstract}

\section{INTRODUCTION}

Supply chain operations have become more complex. Due to factors such as increasing competition and regulations, there has been an increasing need to cover more stages in a supply chain when making a management decision. As more supply chain stages are considered when making decisions, the complexity of the problem increases. One such example is when we want to optimise the combined operations covering both inventory and vehicle routing operations. This problem is widely known as the Inventory-Routing Problem (IRP). IRP is an optimization problem that aims at minimizing the total cost associated with the inventory and vehicle routing operations of a supply chain, thus providing a holistic perspective to the enhancement of the supply chain performance. To enable us to solve a complex IRP, we often need to simplify the problem so that it can be solved efficiently using exact methods or metaheuristics. One common simplification is to assume all inputs to be deterministic. Hence, it is not surprising that most of the early works on IRP assume that the model inputs (e.g., customers' demands, travel costs, etc.) are 


\section{Onggo, Juan, Panadero, Corlu, and Agustin}

deterministic (Coelho et al. 2013). Hence, these works tend to analyze optimization models that oversimplify the uncertainty that characterizes real-life supply chain operations. As this paper will demonstrate, this 'deterministic assumption' is risky, and might lead to misleading conclusions on the total cost of the supply chain operations, which might have a noticeable impact on the supply chain performance.

IRP is an extended version of the well-known vehicle routing problem (VRP) (Faulin and Juan 2008; Juan et al. 2009). Hence, it makes IRP an NP-hard optimization problem, which limits the practical application of exact methods to small-sized instances. This justifies the use of heuristic-based solving approaches. This paper proposes the use of simulation-optimization methods as an effective way to address stochastic optimization problems (De Armas et al. 2017; Gonzalez-Martin et al. 2018). In particular, the paper uses a simheuristic algorithm - which combines simulation with heuristics - to generate high-quality solutions to stochastic IRPs. A recent review on the simheuristics concept and its applications can be found in Juan et al. (2018).

We illustrate with some numerical examples why optimization results based on the assumption of deterministic inputs might lead to misleading conclusions. To demonstrate this, we consider an IRP with stochastic demand. The planning in this supply chain is composed of two stages: $(i)$ the inventory management at each of the supply chain distribution centers (DCs); and (ii) the transportation of products from a central warehouse to these DCs. The objective is to minimize the total expected cost (including both routing and inventory costs). Simheuristics have an added benefit because they can provide other key performance indicators such as the variability of the cost associated with the proposed operations plan. Since IRP considers the two stages in an integrated way, it is strongly related to the concept of Vendor Managed Inventories (VMI) in supply chain management, where decisions on inventory management are transferred to the central supplier. A discussion of the VMI concept, including the use of simulation models, can be found in Marquès et al. (2010).

Figure 1 represents a simple example of an IRP. A product is distributed from a central warehouse to a series of DCs. Each DC manages an inventory to supply an aggregated customer's demand, which follows a best-fit probability distribution based on historical data. Each DC has an initial stock level, which is assumed to be known. While the IRP is often formulated as a multi-period problem, in this work we will consider the single-period version with random demands and possible stock-outs at each DC. These stock-outs might occur whenever the actual aggregate demand at any DC is higher than its expected value and there is not enough safety stock at the DC to cover it. Hence, several authors have considered stock-outs when modeling the IRP, among them Trudeau and Dror (1992) and Bertazzi et al. (2013). Whenever a stock-out occurs, a penalty cost is applied to 'repair' the failure in the operations plan. In our case, this penalty cost is given by a direct shipment to the DC where the stock-out occurred (i.e., the penalty cost of a stock-out is equivalent to the cost of a round-trip from the warehouse to that DC).

The remainder of this paper is structured as follows: existing literature on the stochastic IRP is reviewed in Section 2; more details on the particular problem being addressed are provided in Section 3; Section 4 outlines our simulation-optimization approach for dealing with the IRP with stochastic demands and stockouts; in Section 5, a complete set of experiments is described and analyzed; finally, Section 6 highlights the main contributions of this work.

\section{RELATED WORK}

The deterministic version of the IRP has been explored in many studies. However, this is not the case for the stochastic version of the problem. Random demands for inventory routing over an infinite horizon is addressed in Jaillet et al. (2002), who present incremental cost approximations in a rolling horizon framework. The stochastic IRP is formulated as a Markov decision process by Adelman (2004). The author applies cost approximations by using dual prices of a linear program. Further approximation methods for the IRP with demand uncertainty modeled as a Markov decision problem are discussed by Kleywegt et al. (2004). Another approach in which the stochastic IRP is modeled as a Markov decision process is presented in Hvattum et al. (2009). The authors model random demand through general discrete distributions, while 


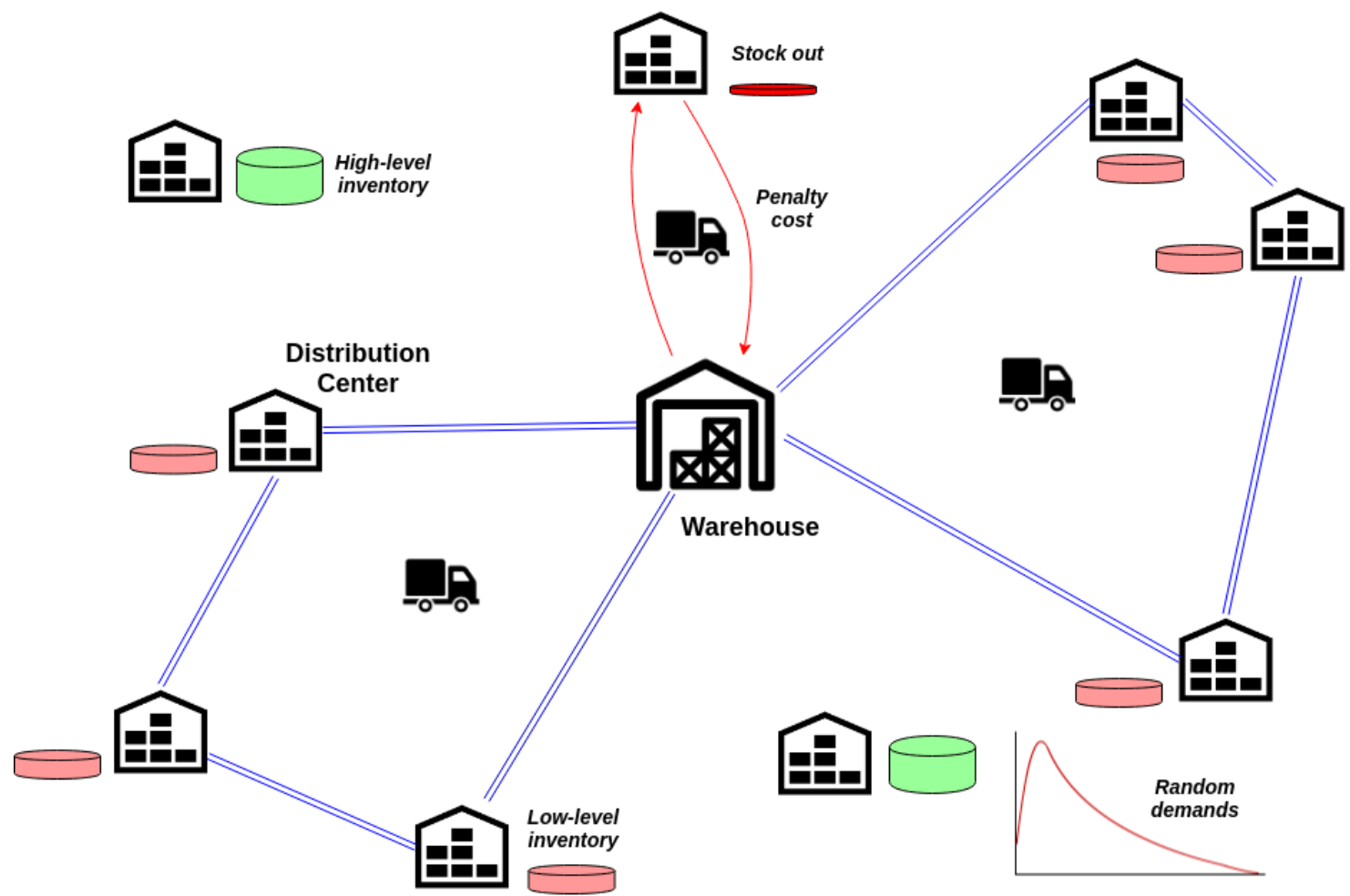

Figure 1: A simple example of the inventory routing problem.

their solution framework is based on the use of scenario trees. Solutions to the scenario tree problem are obtained by using a standard mixed-integer linear programming (MILP) solver, a greedy randomized adaptive search procedure, and a progressive hedging algorithm.

More recent work on stochastic inventory routing include the one of Bertazzi et al. (2013), who consider an IRP with stock outs and a finite horizon, solved with a dynamic programming model and a hybrid roll-out algorithm. Bertazzi et al. (2015) address the IRP with stochastic demand and transportation procurement by developing a roll-out-based matheuristic algorithm. A similar problem is addressed in a robust optimization approach through MILP formulations by Soysal et al. (2015), who propose a robust-based approach which assumes a uniform random behavior for these demands. These authors include environmental concerns in their solution for the IRP with demand uncertainty by estimating pollutant emissions in the route planning process.

Huang and Lin (2010) develop a modified ant colony optimization metaheuristic for the multi-product IRP with demand uncertainty. A robust inventory routing policy, considering stochastic customer demands and replenishment lead-times, is discussed by Li et al. (2016). The robustness of inventory replenishment and customer selection policies for the dynamic and stochastic IRP is addressed in Roldán et al. (2016). Yu et al. (2012) solve the stochastic IRP with split deliveries and service level constraints.

Greenhouse gas emissions are also minimized in the work of Niakan and Rahimi (2015), who propose a fuzzy possibilistic approach to a multi-objective IRP for medical drug distribution. Abdul Rahim et al. (2014) solve the multi-period IRP with stochastic stationary demand through a deterministic equivalent approximation model. Also addressing the IRP with stochastic demands, Chen and Lin (2009) consider a real life multi-period, multi-product case. In addition, the authors incorporate risk aversion into their model. More closely related to our work, Juan et al. (2014) propose a simheuristic approach, combining a multi-start metaheuristic with Monte Carlo simulation, to address the single-period IRP with stochastic demands. An enhanced simheuristic is proposed in Gruler et al. (2018a), who employ an algorithm based on the variable neighborhood search framework to solve a single-period IRP. Finally, Gruler et al. (2018b) 
extend their previous approach to the multi-period IRP by incorporating a special constructive component in their simheuristic.

\section{ADDITIONAL DETAILS OF THE CONSIDERED PROBLEM}

As explained in Section 1, the IRP combines inventory-management and vehicle-routing decisions. Let us denote by $V=\{0,1, \ldots, n\}$ the set composed of the central warehouse (node 0 ), and $n$ DCs (nodes 1 to $n$ ). The operational plan is composed of the following sequential stages:

1. Configuration of the replenishment levels and the quantities to be delivered to each DC: In this stage, the decision variable $r_{i}$, where $r_{i} \in\left[0, \hat{l}_{i}\right]$, is the target replenishment level at customer $i$, and the maximum capacity of DC $i$ is $\hat{l}_{i}(\forall i \in V \backslash\{0\})$. The chosen replenishment level directly affects the order quantity $q_{i} \geq 0$ to be delivered from the warehouse to DC $i$, which can be computed as a function of target replenishment level $r_{i}$ and the current stock level $l_{i} \in\left[0, \hat{l}_{i}\right]$, as expressed in Equation (1):

$$
q_{i}=q_{i}\left(r_{i}, l_{i}\right)=\left\{\begin{array}{lll}
r_{i}-l_{i} & \text { if } \quad l_{i}<r_{i} \\
0 & \text { if } \quad l_{i} \geq r_{i}
\end{array} \quad \forall i \in V \backslash\{0\} .\right.
$$

2. Configuration of the routing plan and computation of the routing cost: In this stage, a minimum-cost routing plan is designed to deliver the $q_{i}$ assigned to each DC $i$. Notice that whenever $q_{i}=0$ for some $i \in V \backslash\{0\}$, then the corresponding DC will not be visited by any vehicle (route) to avoid incurring unnecessary routing costs. As described in Faulin et al. (2008), we assume that a fleet of homogeneous vehicles, each with a limited loading capacity, is available at the warehouse. Let $A=\{(i, j) \mid i, j \in V, i \neq j\}$ be the set of edges linking nodes in $V$, and $c_{i j}=c_{j i}>0$ the travel cost associated with edge $(i, j) \in A$. Let $x_{i j}^{k}$ be a binary variable that takes the value 1 if edge $(i, j)$ is traversed by a route $k$ and 0 otherwise. Then, the overall routing costs $R$ can be expressed as in Equation (2):

$$
R=\sum_{(i, j) \in A} \sum_{k \in K} c_{i j} \cdot x_{i j}^{k} .
$$

3. Realization of random demands and computation of the associated inventory costs: In this stage, random observations $d_{i} \geq 0$ of the stochastic customers' demands $D_{i}$ at each DC $i$ are obtained. Depending on the exact value of these realizations, holding-inventory or stock-out costs, $f_{i} \geq 0$, is generated as described in Equation (3):

$$
f_{i}=f_{i}\left(q_{i}, l_{i}, d_{i}\right)=\left\{\begin{array}{lll}
\lambda_{i}\left(q_{i}+l_{i}-d_{i}\right) & \text { if } & q_{i}+l_{i} \geq d_{i} \\
2 \cdot c_{0 i} & \text { if } & q_{i}+l_{i}<d_{i}
\end{array} \quad \forall i \in V \backslash\{0\} .\right.
$$

where $\lambda_{i}$ represents the unitary holding cost at DC $i$ and $c_{0 i}$ represents the cost of a one-way trip from the warehouse to DC $i$ (i.e., whenever a stock-out occurs at DC $i$, the extra cost of a round-trip from the depot to the DC has to be considered as a costly but necessary 'repair' action). Notice that the expected inventory cost $E[I]$ is given by Equation (4):

$$
E[I]=E\left[I\left(q_{i}, l_{i}, D_{i} ; \forall i \in V \backslash\{0\}\right)\right]=\sum_{i \in V \backslash\{0\}} E\left[f_{i}\left(q_{i}, l_{i}, D_{i}\right)\right] .
$$

The sum of Equations (2) and (4) represents the total expected cost of the operations plan. Our first goal then is to generate IRP solutions with a low total expected cost. However, we will also take a look at the variance of these solutions. The reason is that a plan that minimizes the total expected cost but shows a high variability in the cost value due to variations in the values associated with the random demands 


\section{Onggo, Juan, Panadero, Corlu, and Agustin}

might be too risky for the decision maker; hence, the plan might be far from being 'optimal' in a scenario with input uncertainty.

\section{A SIMULATION-OPTIMIZATION METHOD}

In this section we describe our simheuristic approach to solve the single-period IRP with stochastic demands and stock-outs. Simheuristics combine heuristics with simulation (in any of its forms). This hybridization has been used to extend metaheuristic frameworks, such as the greedy randomized adaptive search procedure (Ferone et al. 2019), the iterated local search (Grasas et al. 2016), or the large neighborhood search (Dominguez et al. 2016) so they can deal with stochastic optimization problems.

One of the main ideas behind our approach is to consider a single period and assumes that updated information on current inventory levels is obtained at the end of each period. The end-of-period inventory levels might be very difficult to forecast, especially when the variance of the random demands is high. Hence, we plan for the period ahead and then update the current inventory levels before planning again. Similarly, adjustments on the probability distributions that represent customers' demands can be made at the beginning of a new period in order to include trend and seasonal factors. Thus, our planning methodology can be iteratively employed at the beginning of each period, using updated inputs whenever they become available. It is assumed that the central warehouse has enough stock to satisfy the quantities that need to be delivered to each DC in each period.

As illustrated in Figure 2, our approach in solving this IRP problem comprises the following stages:

1. In the first stage, Monte Carlo simulation is employed to estimate, for each DC, the expected inventory cost associated with each of the considered policies. As explained in the previous section, these inventory costs include both inventory surplus as well as penalty costs generated by inventory stock-outs. Although more intermediate policies could be employed if necessary, in this paper, the following replenishment levels are considered, where each value represents a given percentage of the total DC capacity: $0 \%$ (no refill), 25\%, 50\%, 75\%, and 100\% (full refill). Then, the replenishment level of each DC is set to the one that minimizes the expected inventory cost and a biased-randomized heuristic (Grasas et al. 2017) is employed to generate an initial routing plan.

2. In the second stage, a multi-start procedure is carried out. In each iteration of this procedure, the following steps are executed over the initial configuration of policies derived in the first stage: $(i)$ the list of DCs is randomly sorted using a uniform distribution; (ii) for each DC in the list, each of its replenishment policies is tested, and for each policy the aforementioned biased-randomized heuristic is run to find out the configuration with the best total - inventory plus routing - cost; and (iii) a list with the top policy-configuration maps is stored.

3. In the third stage, each of the 'elite' policy-configuration maps selected in the previous stage are sent throughout a more intensive vehicle routing algorithm in order to enhance its associated routing cost. In our case, a parallelized version of our biased-randomized heuristic is employed (Fikar et al. 2016; Martin et al. 2016). Also, a risk analysis is performed in order to estimate the variance of the cost associated with each operations plan.

\section{COMPUTATIONAL EXPERIMENTS}

In order to test our method, we employ the IRP benchmark proposed by Juan et al. (2014). This benchmark generalizes the well-known datasets $A$ and $B$ from the vehicle routing problem literature (Augerat et al. 1998). These datasets consist of 27 small- and medium-sized test instances, with the number of nodes ranging from 32 to 80 (each node represents a DC). The associated deterministic demands are considered to be the expected values of the new random demands $D_{i}, \forall i \in V \backslash\{0\}$. For the numerical experiments of this paper, we will assume that $D_{i}$ follows a LogNormal probability distribution. As discussed in Cobb et al. (2013), this distribution is frequently used to model non-negative random variables, such as continuous demands 


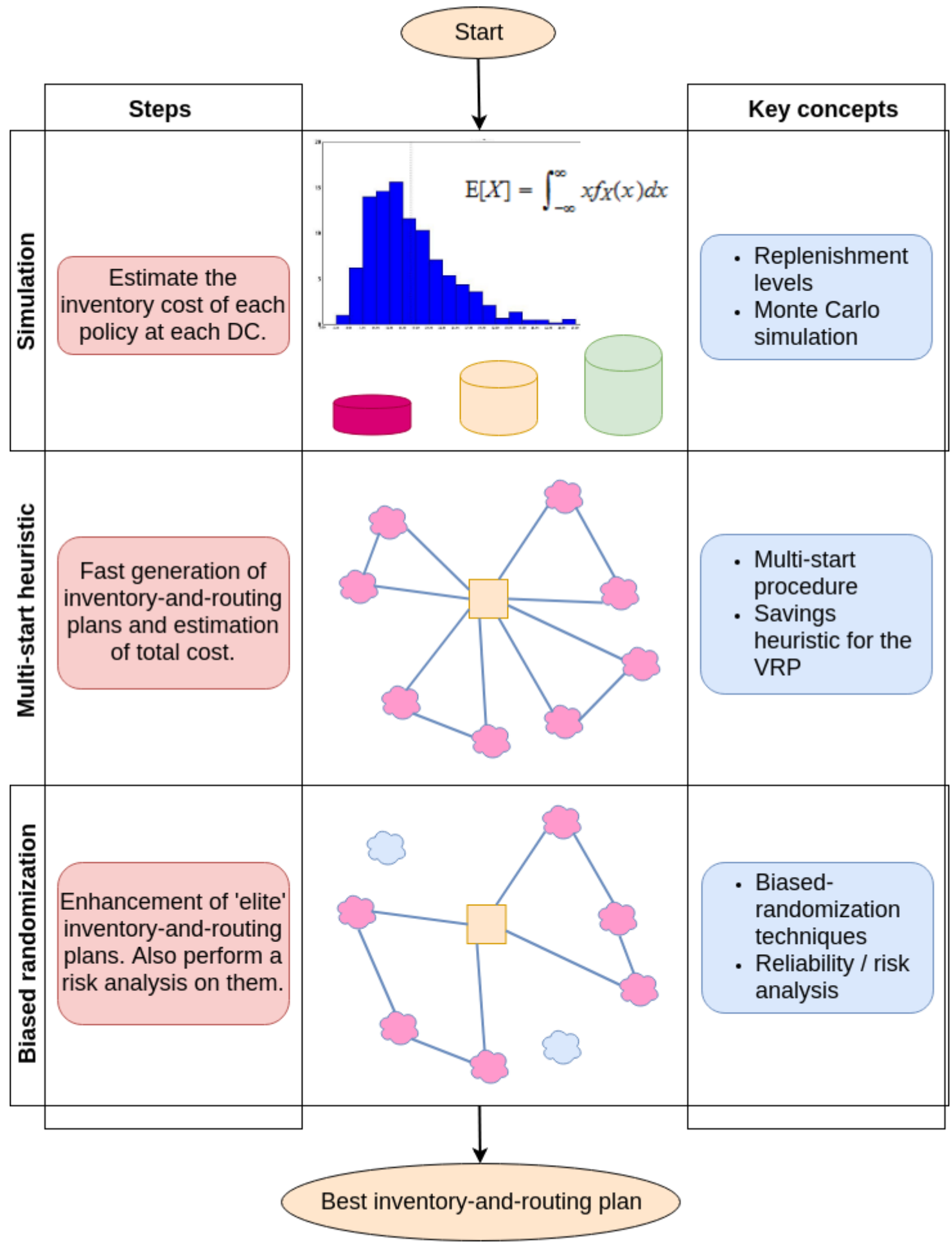

Figure 2: Visual overview of our simheuristic approach.

or times. Notice that our simheuristic approach could also employ any other probability distribution that fits the available historical data. We also consider four different levels of variance, i.e., $\operatorname{Var}\left[D_{i}\right]=c \cdot E\left[D_{i}\right]$, 


\section{Onggo, Juan, Panadero, Corlu, and Agustin}

with $c=0$ defining a 'deterministic' scenario, $c=0.25$ defining a 'low-variance' scenario, $c=0.5$ defining an 'intermediate-variance' scenario, and $c=0.75$ defining a 'high-variance' scenario.

The method described in the previous section has been implemented as a Java application and used to run the VRP instances described above for each combination of the parameter $k$ and using $\lambda=0.5$. Each of these tests was run for a maximum computing time of 10 seconds on a standard personal computer. On top of this, we need to estimate the inventory costs for each DC-policy combination; each of which requires one million simulation runs and takes about 1.5 seconds.

Tables 1 to 4 show the results obtained using our approach. The first column shows the instance name. The second column shows the best-known solution (BKS) as provided in Juan et al. (2014). The next two columns display the costs associated with the inventory management and the routing planning. The total cost is given next, as well as the percentage gap with respect to the BKS. Notice that for all instances and variance levels, the simheuristic approach introduced in this paper is able to outperform the results provided in the literature. This is mainly due to the addition of the last refinement stage as well as to the use of a more efficient routing procedure. As expected, the performance of simheuristic is affected by the variance level. A higher variance level makes the problem more unpredictable (i.e. the realisation of the demand has a wider range). Hence, it increases the number of stock-outs which leads to higher stock-out cost and total inventory cost (column 3 in the tables).

Tables 1 to 4 also illustrate the risk of ignoring input uncertainty when making management decisions. Let us consider a case where the real demand is stochastic with variance $c=0.25$. If we use a model that assumes the demand is deterministic, the associated distribution plan will not protect itself against the risk of possible variations in customers' demands. As a consequence, expensive stock-outs might occur, thus increasing the total cost of managing inventories. The difference in the inventory cost (column 3 ) in tables 1 and 2 shows the magnitude of the underestimation (i.e. on average the real inventory cost is more than double). The magnitude is greater as the variance increases (e.g. if the real demand is stochastic with variance $c=0.75$, the real inventory cost is more than four times of the estimate from the deterministic model).

Table 1: Results for a variance level equal to 0 (deterministic).

\begin{tabular}{llrrrr}
\cline { 3 - 5 } & \multicolumn{5}{c}{ OBS $(c=0.00)$} \\
\hline Instance Name & BKS (1) & Stock Costs (2) & Routing Costs (3) & Total Costs (4) & Gap (\%) [1-4] \\
\hline A-n37-k5 & 529.58 & 48.93 & 457.72 & 506.65 & -4.33 \\
A-n38-k5 & 560.19 & 35.76 & 502.29 & 538.06 & -3.95 \\
A-n39-k6 & 555.21 & 58.46 & 484.53 & 542.99 & -2.20 \\
A-n45-k6 & 688.08 & 44.13 & 635.94 & 680.07 & -1.16 \\
A-n45-k7 & 789.64 & 46.88 & 700.47 & 747.34 & -5.36 \\
A-n55-k9 & 754.14 & 49.39 & 700.41 & 749.80 & -0.58 \\
A-n60-k9 & 900.05 & 53.39 & 842.83 & 896.22 & -0.43 \\
A-n63-k9 & 1065.27 & 67.87 & 958.30 & 1026.17 & -3.67 \\
A-n65-k9 & 742.75 & 61.52 & 677.86 & 739.38 & -0.45 \\
\hline Average: & 731.66 & 51.81 & 662.26 & 714.07 & -2.46
\end{tabular}

Finally, for the instance A-n38-k5, Figure 3 displays the distribution of the inventory and routing costs associated with different refill strategies, including 0\% refill, $25 \%$ refill, $50 \%$ refill, $75 \%$ refill, and $100 \%$ refill. One can notice that when no refill is assigned ( $0 \%$ refill strategy), all costs are due to inventory management, which also includes potential stock-outs. Likewise, as higher refill levels are considered, inventory costs tend to decrease while routing costs tend to increase. One can also notice that significant reductions in total cost can be achieved by implementing our methodology, since our best solution (OBS) shows a much lower total cost than other refill strategies. 
Onggo, Juan, Panadero, Corlu, and Agustin

Table 2: Results for a 'low' variance level.

\begin{tabular}{lr|rrrr}
\cline { 3 - 5 } & & \multicolumn{5}{c}{ OBS $(c=0.25)$} \\
\hline Instance Name & BKS (1) & Stock Costs (2) & Routing Costs (3) & Total Costs (4) & Gap (\%) [1-4] \\
\hline A-n37-k5 & 604.90 & 93.66 & 504.02 & 597.69 & -1.19 \\
A-n38-k5 & 667.65 & 129.44 & 523.97 & 653.41 & -2.13 \\
A-n39-k6 & 764.36 & 107.38 & 636.23 & 743.62 & -2.71 \\
A-n45-k6 & 912.32 & 88.97 & 765.99 & 854.96 & -6.29 \\
A-n45-k7 & 1098.56 & 95.80 & 952.50 & 1048.30 & -4.57 \\
A-n55-k9 & 1085.09 & 244.57 & 832.25 & 1076.83 & -0.76 \\
A-n60-k9 & 1280.69 & 133.24 & 1107.93 & 1241.17 & -3.09 \\
A-n63-k9 & 1423.35 & 143.08 & 1276.92 & 1420.00 & -0.24 \\
A-n65-k9 & 1054.87 & 138.29 & 910.35 & 1048.64 & -0.59 \\
\hline Average: & 987.98 & 130.49 & 834.46 & 964.96 & -2.40
\end{tabular}

Table 3: Results for a 'medium' variance level.

\begin{tabular}{lr|rrrr}
\cline { 3 - 5 } & & \multicolumn{4}{c}{ OBS $(c=0.50)$} \\
\hline Instance Name & BKS (1) & Stock Costs (2) & Routing Costs (3) & Total Costs (4) & Gap (\%) [1-4] \\
\hline A-n37-k5 & 643.53 & 131.28 & 512.25 & 640.84 & -0.42 \\
A-n38-k5 & 706.23 & 168.02 & 538.20 & 691.99 & -2.02 \\
A-n39-k6 & 794.24 & 153.15 & 641.09 & 781.64 & -1.59 \\
A-n45-k6 & 932.87 & 139.78 & 793.10 & 920.68 & -1.31 \\
A-n45-k7 & 1140.68 & 137.92 & 1002.76 & 1110.01 & -2.69 \\
A-n55-k9 & 1146.30 & 305.78 & 840.53 & 1140.64 & -0.49 \\
A-n60-k9 & 1337.08 & 212.62 & 1124.46 & 1326.40 & -0.80 \\
A-n63-k9 & 1524.09 & 234.43 & 1289.67 & 1498.09 & -1.71 \\
A-n65-k9 & 1123.30 & 206.71 & 916.58 & 1110.55 & -1.13 \\
\hline Average: & 1038.70 & 187.74 & 850.96 & 1024.54 & -1.35
\end{tabular}

Table 4: Results for a 'high' variance level.

\begin{tabular}{lr|rrrr}
\cline { 3 - 6 } & \multicolumn{5}{c}{ OBS (c=0.75) } \\
\hline Instance Name & BKS (1) & Stock Costs (2) & Routing Costs (3) & Total Costs (4) & Gap (\%) [1-4] \\
\hline A-n37-k5 & 676.86 & 163.68 & 513.17 & 666.32 & -1.56 \\
A-n38-k5 & 799.05 & 131.22 & 667.83 & 794.59 & -0.56 \\
A-n39-k6 & 865.00 & 164.80 & 700.20 & 858.62 & -0.74 \\
A-n45-k6 & 989.00 & 186.04 & 802.95 & 983.22 & -0.58 \\
A-n45-k7 & 1206.54 & 202.87 & 1003.66 & 1185.08 & -1.78 \\
A-n55-k9 & 1198.39 & 346.23 & 852.16 & 1174.75 & -1.97 \\
A-n60-k9 & 1413.71 & 287.40 & 1126.31 & 1402.85 & -0.77 \\
A-n63-k9 & 1639.33 & 352.91 & 1286.42 & 1614.91 & -1.49 \\
A-n65-k9 & 1211.51 & 288.95 & 922.57 & 1201.50 & -0.83 \\
\hline Average: & 1111.04 & 236.01 & 875.03 & 1097.98 & -1.14
\end{tabular}




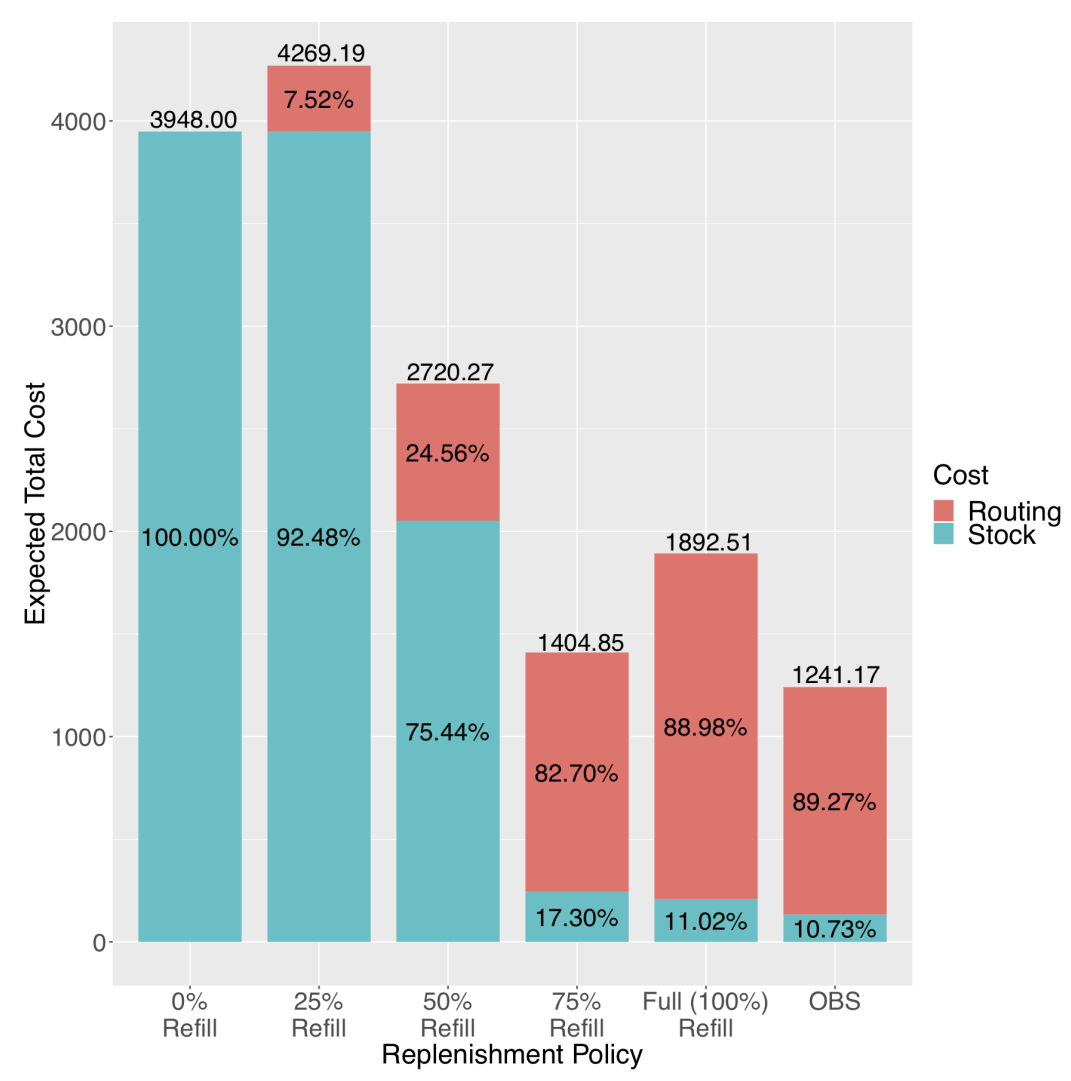

Figure 3: A-n38-k5 average routing and inventory costs for different replenishment strategies.

\section{CONCLUSIONS}

Supply chain management has become more complex. One of the complex supply chain problems is shown in a problem called the Inventory-Routing Problem (IRP) that combines two critical stages of most supply chains: inventory management at each distribution center and product delivery from a central warehouse to these distribution centers. As supply chain has become more complex, the application of exact methods to solve a supply chain optimisation problem has become more limited to small size problems. Hence, there is a need for heuristic methods. In this paper, we have proposed a three-stage simheuristic approach to solve a stochastic IRP. The first stage uses simulation to estimate the inventory cost associated with each possible policy at each distribution center (considering both random demands and the cost of stock-outs). Then, in the second stage, a multi-start heuristic uses the information from the first stage to quickly generate many inventory-and-routing policies, while estimating the associated total cost of each policy. Finally, in the third stage, a refinement step is applied to the best policy obtained from the second stage in order to reduce the routing costs even further. We have evaluated our method using a single-period IRP with stochastic demands at each distribution center taking into account the possibility of stock-outs. Our experiment has shown that our proposed method produces results that are better than the best-known solutions published in scientific literature. We have also demonstrated that when making planning decisions for supply chain operations, ignoring stochasticity in the supply chain can significantly underestimate the total cost. This can lead to wrong decisions.

The following are some of the limitations of our model and solving approach, which also constitute open research opportunities: (i) to take into account the correlations among the random demands at each distribution center in the model, as this is common in some supply chains; (ii) to consider more sources of uncertainty in the supply chain (e.g. processing times, travel times, breakdowns) and how they affect the 


\section{Onggo, Juan, Panadero, Corlu, and Agustin}

model outputs; (iii) to incorporate the quantification of input uncertainty into the simheuristic methodology; and (iv) to implement our method in a symbiotic simulation (Onggo et al. 2018) or a learnheuristic environment (Calvet et al. 2016), so that the model can learn from the real world over time.

\section{ACKNOWLEDGEMENTS}

This work has been partially funded by the IoF2020 project of the European Union (grant agreement no. 731884) and by the Erasmus+ Program (2018-1-ES01-KA103-049767).

\section{REFERENCES}

Abdul Rahim, M. K. I., Y. Zhong, E.-H. Aghezzaf, and T. Aouam. 2014. "Modelling and Solving the Multiperiod Inventory-Routing Problem with Stochastic Stationary Demand Rates". International Journal of Production Research 52(14):4351-4363.

Adelman, D. 2004. "A Price-Directed Approach to Stochastic Inventory/Routing”. Operations Research 52(4):499-514.

Augerat, P., J.-M. Belenguer, E. Benavent, A. Corbéran, and D. Naddef. 1998. "Separating Capacity Constraints in the CVRP using Tabu Search". European Journal of Operational Research 106(2-3):546-557.

Bertazzi, L., A. Bosco, F. Guerriero, and D. Lagana. 2013. "A Stochastic Inventory Routing Problem with Stock-Out". Transportation Research Part C: Emerging Technologies 27:89-107.

Bertazzi, L., A. Bosco, and D. Laganà. 2015. "Managing Stochastic Demand in an Inventory Routing Problem with Transportation Procurement". Omega 56:112-121.

Calvet, L., A. Ferrer, M. I. Gomes, A. A. Juan, and D. Masip. 2016. "Combining Statistical Learning with Metaheuristics for the Multi-Depot Vehicle Routing Problem with Market Segmentation”. Computers \& Industrial Engineering 94:93-104.

Chen, Y. M. and C.-T. Lin. 2009. "A Coordinated Approach to Hedge the Risks in Stochastic Inventory-Routing Problem". Computers \& Industrial Engineering 56(3):1095-1112.

Cobb, B. R., R. Rumí, and A. Salmerón. 2013. "Inventory Management with Log-normal Demand per Unit Time”. Computers \& Operations Research 40(7):1842-1851.

Coelho, L. C., J.-F. Cordeau, and G. Laporte. 2013. "Thirty Years of Inventory Routing”. Transportation Science 48(1):1-19.

De Armas, J., A. A. Juan, J. M. Marquès, and J. P. Pedroso. 2017. "Solving the Deterministic and Stochastic Uncapacitated Facility Location Problem: From a Heuristic to a Simheuristic". Journal of the Operational Research Society 68(10):1161-1176.

Dominguez, O., D. Guimarans, A. A. Juan, and I. de la Nuez. 2016. "A Biased-Randomised Large Neighbourhood Search for the Two-Dimensional Vehicle Routing Problem with Backhauls”. European Journal of Operational Research 255(2):442-462.

Faulin, J., M. Gilibert, A. A. Juan, X. Vilajosana, and R. Ruiz. 2008. "SR-1: A Simulation-based Algorithm for the Capacitated Vehicle Routing Problem”. In Proceedings of the 2008 Winter Simulation Conference, edited by S. J. Mason, R. R. Hill, L. Moench, O. Rose, T. Jefferson, and J. W. Fowler, 2708-2716. Piscataway, New Jersey: Institute of Electrical and Electronics Engineers, Inc.

Faulin, J. and A. A. Juan. 2008. "The ALGACEA-1 Method for the Capacitated Vehicle Routing Problem". International Transactions in Operational Research 15(5):599-621.

Ferone, D., A. Gruler, P. Festa, and A. A. Juan. 2019. "Enhancing and Extending the Classical GRASP Framework with Biased Randomisation and Simulation". Journal of the Operational Research Society 70(8):1362-1375.

Fikar, C., A. A. Juan, E. Martinez, and P. Hirsch. 2016. "A Discrete-Event Driven Metaheuristic for Dynamic Home Service Routing with Synchronised Trip Sharing". European Journal of Industrial Engineering 10(3):323-340.

Gonzalez-Martin, S., A. A. Juan, D. Riera, M. G. Elizondo, and J. J. Ramos. 2018. "A Simheuristic Algorithm for Solving the Arc Routing Problem with Stochastic Demands". Journal of Simulation 12(1):53-66.

Grasas, A., A. A. Juan, J. Faulin, J. de Armas, and H. Ramalhinho. 2017. "Biased Randomization of Heuristics using Skewed Probability Distributions: A Survey and some Applications". Computers \& Industrial Engineering 110:216-228.

Grasas, A., A. A. Juan, and H. R. Lourenço. 2016. "SimILS: A Simulation-Based Extension of the Iterated Local Search Metaheuristic for Stochastic Combinatorial Optimization”. Journal of Simulation 10(1):69-77.

Gruler, A., J. Panadero, J. de Armas, J. A. Moreno, and A. A. Juan. 2018a. "Combining Variable Neighborhood Search with Simulation for the Inventory Routing Problem with Stochastic Demands and Stock-Outs". Computers \& Industrial Engineering 123:278-288.

Gruler, A., J. Panadero, J. de Armas, J. A. Moreno, and A. A. Juan. 2018b. "A Variable Neighborhood Search Simheuristic for the Multiperiod Inventory Routing Problem with Stochastic Demands". International Transactions in Operational Research 27(1):314-335.

Huang, S.-H. and P.-C. Lin. 2010. "A Modified Ant Colony Optimization Algorithm for Multi-Item Inventory Routing Problems with Demand Uncertainty". Transportation Research Part E: Logistics and Transportation Review 46(5):598-611.

Hvattum, L. M., A. Løkketangen, and G. Laporte. 2009. "Scenario Tree-Based Heuristics for Stochastic Inventory-Routing Problems". INFORMS Journal on Computing 21(2):268-285. 


\section{Onggo, Juan, Panadero, Corlu, and Agustin}

Jaillet, P., J. F. Bard, L. Huang, and M. Dror. 2002. "Delivery Cost Approximations for Inventory Routing Problems in a Rolling Horizon Framework". Transportation Science 36(3):292-300.

Juan, A. A., J. Faulin, R. Ruiz, B. Barrios, M. Gilibert, and X. Vilajosana. 2009. "Using Oriented Random Search to Provide a Set of Alternative Solutions to the Capacitated Vehicle Routing Problem”. In Operations Research and Cyber-Infrastructure, 331-345. Springer.

Juan, A. A., S. E. Grasman, J. Caceres-Cruz, and T. Bektaş. 2014. "A Simheuristic Algorithm for the Single-Period Stochastic Inventory-Routing Problem with Stock-Outs". Simulation Modelling Practice and Theory 46:40-52.

Juan, A. A., W. D. Kelton, C. S. Currie, and J. Faulin. 2018. "Simheuristics Applications: Dealing with Uncertainty in Logistics, Transportation, and other Supply Chain Areas". In Proceedings of the 2018 Winter Simulation Conference, edited by M. Rabe, A. A. Juan, N. Mustafee, A. Skoogh, S. Jain, and B. Johansson, 3048-3059. Piscataway, New Jersey: Institute of Electrical and Electronics Engineers, Inc.

Kleywegt, A. J., V. S. Nori, and M. W. Savelsbergh. 2004. "Dynamic Programming Approximations for a Stochastic Inventory Routing Problem”. Transportation Science 38(1):42-70.

Li, M., Z. Wang, and F. T. Chan. 2016. "A Robust Inventory Routing Policy under Inventory Inaccuracy and Replenishment Lead-Time". Transportation Research Part E: Logistics and Transportation Review 91:290-305.

Marquès, G., C. Thierry, J. Lamothe, and D. Gourc. 2010. "A Review of Vendor Managed Inventory (VMI): From Concept to Processes". Production Planning \& Control 21(6):547-561.

Martin, S., D. Ouelhadj, P. Beullens, E. Ozcan, A. A. Juan, and E. K. Burke. 2016. "A Multi-Agent Based Cooperative Approach to Scheduling and Routing". European Journal of Operational Research 254(1):169-178.

Niakan, F. and M. Rahimi. 2015. "A Multi-Objective Healthcare Inventory Routing Problem: A Fuzzy Possibilistic Approach". Transportation Research Part E: Logistics and Transportation Review 80:74-94.

Onggo, B. S. S., N. Mustafee, A. A. Juan, O. Molloy, and A. Smart. 2018. "Symbiotic Simulation System: Hybrid Systems Model Meets Big Data Analytics". In Proceedings of the 2018 Winter Simulation Conference, edited by M. Rabe, A. A. Juan, N. Mustafee, A. Skoogh, S. Jain, and B. Johansson, 1358-1369. Piscataway, New Jersey: Institute of Electrical and Electronics Engineers, Inc.

Roldán, R. F., R. Basagoiti, and L. C. Coelho. 2016. "Robustness of Inventory Replenishment and Customer Selection Policies for the Dynamic and Stochastic Inventory-Routing Problem”. Computers \& Operations Research 74:14-20.

Soysal, M., J. M. Bloemhof-Ruwaard, R. Haijema, and J. G. van der Vorst. 2015. "Modeling an Inventory Routing Problem for Perishable Products with Environmental Considerations and Demand Uncertainty". International Journal of Production Economics 164:118-133.

Trudeau, P. and M. Dror. 1992. "Stochastic Inventory Routing: Route Design with Stockouts and Route Failures". Transportation Science 26(3):171-184.

Yu, Y., C. Chu, H. Chen, and F. Chu. 2012. "Large Scale Stochastic Inventory Routing Problems with Split Delivery and Service Level Constraints”. Annals of Operations Research 197(1):135-158.

\section{AUTHOR BIOGRAPHIES}

BHAKTI STEPHAN ONGGO is an Associate Professor of Business Analytics at the Centre for Operational Research, Management Sciences and Information Systems (CORMSIS), Southampton Business School, University of Southampton, UK. His research interests lie in the areas of predictive analytics using simulation (symbiotic simulation, hybrid modelling, agent-based simulation, discrete-event simulation) with applications in operations and supply chain management and social dynamics (e.g. social risk perception). He is the associate editor for the Journal of Simulation and the chair of The OR Society's Simulation SIG. His email address is b.s.s.onggo@soton.ac.uk.

ANGEL A. JUAN is a Full Professor of Operations Research \& Industrial Engineering in the Computer Science Department at the Universitat Oberta de Catalunya (Barcelona, Spain). He is also the Director of the ICSO research group at the Internet Interdisciplinary Institute and Lecturer at the Euncet Business School. Dr. Juan holds a Ph.D. in Industrial Engineering and an M.Sc. in Mathematics. He completed a predoctoral internship at Harvard University and postdoctoral internships at Massachusetts Institute of Technology and Georgia Institute of Technology. His main research interests include applications of simheuristics and learnheuristics in computational logistics and finance, as well as mathematical e-learning. He has published about 80 articles in JCR-indexed journals and nearly 200 papers indexed in Scopus. His website address is http://ajuanp.wordpress.com and his email address is ajuanp@uoc.edu.

JAVIER PANADERO is an Assistant Professor of Simulation and High Performance Computing in the Computer Science, Multimedia and Telecommunication Department at the Universitat Oberta de Catalunya (Barcelona, Spain). He is also a Lecturer at the Euncet Bussines School, and a member of the ICSO@IN3 research group. He holds a Ph.D. and a M.S. in Computer Science. His major research areas are: high performance computing, modeling and analysis of parallel applications, 


\section{Onggo, Juan, Panadero, Corlu, and Agustin}

and simheuristics. He has co-authored more than 18 articles published in journals and conference proceedings. His website address is http://www.javierpanadero.com and his email address is jpanaderom@uoc.edu.

CANAN GUNES CORLU is an Assistant Professor of Administrative Sciences Department of Metropolitan College at Boston University. She holds a Ph.D. in Operations Management from the Tepper School of Business at Carnegie Mellon University. Her research interest is in the area of the design and analysis of stochastic simulations with applications in operations management. She has published more than 20 articles in journals and conference proceedings. Her recent work on the simulation of inventory systems with unknown input models has been selected as a finalist for INFORMS Minority Issues Forum Best Paper Competition. Her email address is canan@bu.edu.

ALBA AGUSTIN is an Assistant Professor of Operations Research in the Statistics, Computer Science, and Mathematics Department at the Public University of Navarra (Spain). Her research interests include applications of optimization methods in vehicle routing and scheduling. She has published over a dozen peer-reviewed papers in international journals, book chapters, and conference proceedings. She completed an internship at Biccoca University (Italy) and Erasmus internships at the University of Natural Resources and Life Sciences (Austria) and the University of Edinburgh (UK). Her email address is albamaria.agustin@ unavarra.es. 